\title{
Fadiga relacionada ao trabalho de parto: uma proposta de sistematização da assistência de enfermagem
}

Roseléia Maia Santana*, Mariana Torreglosa Ruiz, M.Sc.**, Zaida Aurora Sperli Geraldes Soler, D.Sc.***

* Enfermeira, Aluna do curso de especialização em Enfermagem Obstétrica da Faculdade de Medicina de São José do Rio Preto (FAMERP), ${ }^{* *}$ Enfermeira obstétrica, Universidade de São Paulo (EERP-USP), ***Obstetriz, livre-docente em Enfermagem, docente e orientadora da graduação e da pós-graduação da Faculdade de Medicina de São José do Rio Preto (FAMERP), Coordenadora Geral da Especialização em Enfermagem Obstétrica - FAMERP

\begin{abstract}
Resumo
A fadiga é um fenômeno definido como uma sensação opressiva e sustentada de exaustão e de capacidade diminuída para realizar trabalho físico e mental. É um dos sintomas mais comuns e vivenciados em algum momento na vida das pessoas, porém trata-se de uma queixa subjetiva e multicausal. Desta forma, realizamos revisão da literatura sobre: trabalho de parto, período expulsivo; fadiga e fadiga no ciclo gravídico puerperal. Verificamos que a fadiga é um sintoma comum durante todo o ciclo gravídico-puerperal, atribuído à manifestaçôes fisiológicas da gestação. Entretanto, o sintoma deve ser investigado para descartar causas patológicas. Por fim, propomos a utilização do diagnóstico de enfermagem "fadiga" tal como suas medidas de prevenção e intervenção, durante a assistência ao trabalho de parto. Ressaltamos a escassez de estudos na literatura que abordem o tema, assim como a ausência de estudos que apontem e justifiquem a fadiga no trabalho de parto, o que se mostra freqüente na observação clínica das parturientes. Desta forma, fica clara a necessidade de realização de estudos sobre a temática em questão.
\end{abstract}

Palavras-chave: fadiga, trabalho de parto, cuidados de enfermagem.

\section{Abstract \\ Fatigue related to obstetric labor: a proposal of systematization of the nursing assistance}

Fatigue is a phenomenon defined as an oppressive and sustained feeling of exhaustion and decreased capacity to perform physical and mental work. It is one of the most common symptoms experienced by everybody in his life, but it is a subjective and multicausal complaint. Thus, we review the literature on: labor, expulsive period; fatigue, fatigue during pregnancy and childbirth. Was found that fatigue is a common symptom during the entire pregnancy and postpartum period, attributed to the physiological events of pregnancy. However, the symptom should be investigated to rule out pathological causes. Finally, 
we propose the use of nursing diagnosis of "fatigue" as its measures of prevention and intervention, during labor assistance. We emphasize the lack of studies in the literature as well as the absence of studies that point for fatigue in labor, which is frequently shown in the clinical observation of the parturient. Thus, we need more studies on this subject.

Key-words: fatigue, labor, nursing care.

\section{Resumen}

\section{La fatiga relacionada con el trabajo del parto: una proposición de sistematización de la atención de enfermería}

La fatiga es un fenómeno existente desde la antigüedad, que se define como una sensación de agotamiento y disminución de la capacidad para realizar trabajo físico y mental. Es uno de los síntomas más comunes que las personas padecen en algún momento de la vida, pero es una queja subjetiva y multicausal. Por lo tanto, realizamos una revisión de literatura sobre: trabajo de parto, período expulsivo; fatiga y fatiga durante el ciclo gravídico-puerperal. Verificamos que la fatiga es un síntoma común durante todo el embarazo y el parto, que se atribuye a los eventos fisiológicos del embarazo. Sin embargo, el síntoma debe ser investigado para descartar causas patológicas. Por último, proponemos la utilización del diagnóstico de enfermería "fatiga" así como sus medidas de prevención e intervención, durante la asistencia al trabajo de parto. Hacemos hincapié en la falta de estudios en la literatura que tratan el tema, así como la ausencia de estudios que apunten y justifiquen la fatiga en el trabajo de parto, la cual se muestra frecuente en la observación clínica de las parturientas. Por lo tanto, es evidente la necesidad de estudios sobre el tema en cuestión.

Palabras-clave: fatiga, trabajo de parto, atención de enfermería.

\section{Introdução}

\section{Sistematizaçáo da assistência de enfermagem}

A Sistematização da Assistência de Enfermagem é um procedimento organizado e cientifico que contribui para orientar a prática profissional. Tem como prioridade atender às necessidades humanas básicas de cada indivíduo, sendo que sua característica principal é a avaliação sistemática dos cuidados de enfermagem.

O processo de sistematização consiste nas seguintes fases: levantamento de dados, diagnóstico de enfermagem, planejamento do cuidado, implementação do cuidado e avaliação dos resultados obtidos.

O levantamento de dados visa a identificação de sinais e sintomas apresentados pelo indivíduo. Após essa fase, elaboram-se diagnósticos de enfermagem, que se constituem em julgamentos clínicos das respostas do indivíduo, da família ou da comunidade aos problemas reais e potenciais e aos processos vitais, fornecendo a base para a seleção das intervençóes de enfermagem, buscando atingir os resultados pelos quais o enfermeiro é responsável. O planejamento do cuidado determina a assistên- cia que será prestada ao paciente de acordo com suas necessidades identificadas; a implementação dos cuidados de enfermagem trata da execução do cuidado planejado ao paciente. Por fim, a avaliaçáo dos resultados obtidos consiste na análise diária das açóes implementadas com suas devidas correçôes e adaptações [1,2].

\section{Origem histórica dos diagnósticos de enferma- gem}

O termo diagnóstico de enfermagem teve sua origem na década de 50 , porém, somente na década seguinte, Faye Abdellah apresentou um sistema de classificação para identificação de 21 problemas de enfermagem [3].

A partir daí, iniciou-se um processo de desenvolvimento de classificaçóes e taxonomias para a enfermagem. Durante os anos de 1980 ampliou-se a discussáo sobre a etapa do diagnóstico de enfermagem, bem como o interesse por sua utilização. Os sistemas de classificação existentes foram sendo ampliados, e incorporaram outros termos considerados específicos na prática de enfermagem.

Em 1987, o Grupo Norte-Americano para Classificação dos Diagnósticos de Enfermagem ofi- 
cialmente se tornou a Associação Norte-Americana de Diagnósticos de Enfermagem (NANDA), abrindo às conferências para a participação da comunidade científica da enfermagem [4]. A enfermagem brasileira teve sua primeira participação a partir da $8^{a}$ Conferência, realizada em 1989 [5].

Em 2005, foi publicada uma nova edição dos diagnósticos de enfermagem da NANDA: definiçóes e classificação, constituída de 13 domínios, 46 classes, 167 diagnósticos e sete eixos. Cada domínio é composto por classes, cada classe é composta por diagnósticos. Um eixo tem o propósito de dimensionar e especificar melhor a resposta humana em questáo, ou seja, tem como objetivo de compor o enunciado do diagnóstico de enfermagem especificando com fidedignidade as situaçóes clínicas.

Destacamos que a sistematização da assistência de enfermagem é de fundamental importância no trabalho de parto, uma vez que o enfermeiro obstetra tem possibilidade de analisar e avaliar fatores de risco aos quais a parturiente possa estar exposta $\mathrm{e}$ posteriormente tomar decisóes acerca do problema identificado.

\section{Desenvolvimento}

Buscamos através da revisão da literatura abordar e discutir os seguintes temas abaixo descritos: trabalho de parto e parto; período expulsivo do trabalho de parto; fadiga e fadiga no ciclo gravídicopuerperal.

\section{Trabalho de parto e parto}

O parto é uma experiência extremamente importante na vida de uma mulher. Para Lebovici, o parto é considerado um momento crucial na vida da mulher, por inúmeras razóes. Em primeiro lugar, é o momento em que mãe e bebê vão, finalmente, poder se encontrar frente a frente, também é o momento de se deparar com um produto seu, do seu corpo, que mostrará para o mundo sua capacidade ou incapacidade feminina de gerar uma criança [6].

A experiência de dar à luz é tão marcante que, durante anos, o evento e os sentimentos experimentados durante o nascimento do bebê serão lembrados nos mínimos detalhes [7]. A dor, a ansiedade, o medo são vivenciados por elas de maneira única, e cada uma sente-os em momentos diferentes.
As conversas sobre o parto, entre mulheres próximas e mulheres da família, são transmitidas de geração a geração, associando o trabalho de parto geralmente à experiência dolorosa desencadeando sentimento de medo e ansiedade nas gestantes, que podem dificultar o trabalho de parto posteriormente.

Assim, a dor é vista pela mulher como essencial no processo parturitivo e, ao descrever sua evoluçáo e o comportamento assumido, mostra sentir-se em perigo, ameaçada, porque revela medo diante do desconforto provocado pela dor.

As parturientes acreditam que esse desconforto possa ser superado através de resistência física, e roga pelo parto como um evento que possibilitará o término de seu sofrimento. Desse modo, as dores no momento da expulsão terminam aceitas como o fim da parturição, ou seja, o nascimento de seu filho [8].

Acreditamos que dentre todas as mudanças psicológicas, emocionais e físicas desencadeadas pelo trabalho de parto em curto espaço de tempo, a fadiga tem um papel muito importante, merecendo ser alvo de estudos, a fim de melhor detecção e maiores intervençóes no cuidado.

\section{Período expulsivo}

Classicamente, o parto é dividido em quatro períodos clínicos: período de dilatação (primeiro período do parto); período expulsivo (segundo período); dequitaçáo (terceiro período) e período de Greenberg ou puerpério imediato (quarto período) [9-12].

O período expulsivo inicia-se com dilatação completa da cérvix $(10 \mathrm{~cm})$ e termina com a expulsão do feto. Clinicamente, a parturiente passa por 3 fases neste período, abaixo descritas [10,11]:

- $1^{\text {a }}$ Fase - fase latente: período de repouso; parturiente encontra-se relativamente calma e propensa a receber orientaçóes; relaxa no período entre as contraçóes.

- $2^{\mathrm{a}}$ Fase - descida fetal e reflexo de Ferguson (puxos): liberação de ocitocina na hipófise posterior que provoca contraçóes mais fortes; durante as contraçóes uterinas, o ânus ao se distender, provoca uma sensação defecatória, exercendo assim os puxos, que se trata de contraçóes involuntárias da musculatura no sentido de expelir o feto. 
- $3^{\mathrm{a}}$ Fase - fase de transição: apresentação fetal encontra-se no períneo; dor perineal causada pela pressão da apresentação e distensão da musculatura do períneo. Nesse momento as parturientes relatam sentir muita dor, medo, exaustáo e cansaço, dificultando açóes e orientaçóes.

A dor perineal do período expulsivo provém do estiramento dos tecidos perineais para permitir a passagem do feto e da traçáo sobre o peritônio e sobre os ligamentos uterocervicais durante as contraçóes. Também pode ser provocada pelas forças de expulsão ou pela pressão provocada pela apresentação fetal sobre a bexiga, intestinos e outras estruturas pélvicas sensíveis [13-15].

Segundo Rezende, o medo do parto determina forte tensão, e a tensão persistente e excessiva da musculatura uterina impede o completo relaxamento entre as contraçóes. Essa desarmonia faz do parto um processo doloroso e prolongado, levando ao cansaço e esgotamento da mulher [11].

\section{Fadiga}

Segundo NANDA, fadiga é definida como uma sensação opressiva e sustentada de exaustáo e de capacidade diminuída para realizar trabalho físico e mental no nível habitual [16]. Contudo ainda não existe na literatura consenso sobre a definição do termo fadiga, sendo encontrado informaçóes tal como pesquisas escassas sobre o tema em questão [17].

Entretanto, a fadiga é um fenômeno relatado desde a antiguidade. $\mathrm{O}$ termo é derivado do latim 'fatigäre', e encontra-se descrito na Bíblia relacionando-o a sofrimento por causas físicas (trabalho excessivo) ou mentais (sentimento de culpa) [8].

A fadiga ou excesso de cansaço representa um dos sintomas mais comuns e freqüentemente vivenciados em algum momento na vida das pessoas $[18,19]$. Entretanto, as pessoas náo procuram o serviço de saúde pela simples presença do sintoma isolado, mas a fadiga, geralmente aparece secundariamente na fala de pessoas com problemas de saúde [17]. Trata-se de um fenômeno subjetivo, multicausal, que envolve aspectos tanto de ordem física quanto emocional [19].

Existe uma forte relação entre aspectos psicológicos e presença de fadiga [18], porém dentre os fatores multicausais da fadiga destacamos abaixo [17]:
- Causas relacionadas a hábitos: uso de álcool el ou drogas ilícitas; privação do sono; excesso de trabalho; sedentarismo; hábitos alimentares inadequados (dieta não adequada);

- Causas sociais: excesso de trabalho ou desemprego; situaçóes de estresse;

- Causas psicológicas: ansiedade; depressão; síndrome do estresse pós-traumático; desordens do sono;

- Causas físicas: uso de medicaçóes (por exemplo, anti-hipertensivos); processos infecciosos (exemplo: infecção pelo vírus Epstein-Barr); anemia; insuficiência cardíaca; doenças crônicas debilitantes; neoplasias; hipotireioidismo; fibromialgia; sintomas de fadiga crônica sem causa esclarecida (persistente por mais de 6 meses)

- Causas desconhecidas.

Apesar de ser um sintoma subjetivo, destaca-se que a fadiga deve ser investigada e o profissional de saúde deve sempre acreditar no sintoma relatado e não simplesmente questionar ou minorizar sua existência [17].

\section{Fadiga no ciclo gravídico-puerperal}

Verificamos a partir da revisão da literatura que o diagnóstico de enfermagem fadiga está presente durante o trabalho de parto, mas não se manifesta apenas nas parturientes está presente na maioria das vezes em gestantes e puérperas.

Dentre os resultados encontrados, verificamos que entre 62 a $73 \%$ das gestantes apresentam o diagnóstico [19,20]. As características definidoras mais citadas foram: aumento de queixas físicas; sonolência mesmo que em ambientes barulhentos; cansaço. Dentre os fatores relacionados foram encontrados em ordem de freqüência: sedentarismo; ansiedade e anemia [19]. Ainda encontramos o diagnóstico fadiga implícito na queixa de fraqueza relatada pelas mulheres durante consultas/atendimentos [21,22].

Os autores atribuem a presença da fadiga relacionada ao aumento do volume abdominal secundário ao aumento do volume e estiramento do útero gravídico, levando à dificuldade, tal como paralelamente a ansiedade no terceiro trimestre gestacional, causando cansaço freqüente e conseqüente 
diminuição da energia [20], sendo resultado de um processo de adaptação gravídico.

Entretanto faz-se necessário diferenciar sinais e sintomas das adaptaçôes gravídicas dos sinais e sintomas clínicos de anemia. A anemia caracteriza-se pela presença de palidez cutâneo-mucosa, taquicardia, taquipnéia, cansaço a esforços maiores ou menores dependentes do grau da doença, entre outros. Os autores recomendam realizar análise laboratorial para elucidação diagnóstica na vigência da queixa [23].

Embora, reconheçamos com freqüência, o sintoma fadiga durante o trabalho de parto, não encontramos nenhum trabalho na literatura sobre o tema em questão.

\section{Discussão}

A partir do levantamento obtido a partir da revisão da literatura, propomos o reconhecimento e a utilização do diagnóstico de enfermagem: "Fadiga relacionada ao esforço físico aumentado secundária ao periodo expulsivo do trabalho de parto evidenciada por: aumento da necessidade de repouso; cansaço; verbalização de falta de energia; desatençáo; letargia; introspecção; concentraçâo comprometida" na prática clínica.

Acreditamos que a elaboração e utilização do diagnóstico de enfermagem proposto seja uma ferramenta eficaz para nortear as ações preventivas no sentido de proporcionar conforto e apoio às parturientes, promovendo uma assistência de enfermagem humanizada.

\section{Conclusão}

A fadiga é uma queixa freqüentemente encontrada na população em geral, porém é uma queixa subjetiva e multicausal. A fadiga presente no ciclo gravídico-puerperal se deve principalmente às adaptaçôes gestacionais, porém deve ser investigada cuidadosamente. Desta forma, realizamos uma revisão bibliográfica acerca do tema e propomos a adoção do diagnóstico de enfermagem "fadiga" tal como suas medidas de prevenção e intervenção, durante a assistência ao trabalho de parto. Ressaltamos a escassez de estudos na literatura que abordem o tema, assim como a ausência de estudos que apontem e justifiquem a fadiga no trabalho de parto, o que se mostra freqüente na observação clínica das parturientes.
Desta forma, fica clara a necessidade de realização de estudos sobre a temática em questão.

\section{Referências}

1. Horta WA. Processo de enfermagem. São Paulo: Pedagógica e Universitária da Universidade de São Paulo; 1979.

2. Lacava RMVB, Barros SMO. Diagnósticos de enfermagem na assistência às gestantes. Acta Paul Enferm 2004;17(1):9-17.

3. Abdellah FG, Beland I, Martin A, Matheney RV. Patient-centered approaches in nursing. New York: McMillan; 1960.

4. Gordon M. Clinical diagnosis in health professions. Nursing diagnosis - process and application. 2 ed. New York: McGraw-Hill; 1987. p.7-10.

5. Melo AS. Validação dos diagnósticos de enfermagem disfunção sexual e padróes de sexualidade ineficazes [tese]. São Paulo: Escola de Enfermagem de Ribeirão Preto, Universidade de São Paulo; 2004.

6. Lebovici S. O bebê, a máe e o psicanalista. Porto Alegre: Artes Médicas, 1987.

7. Kitzinger S. A experiência de dar à luz. São Paulo: Martins Fontes; 1987.

8. Simóes SMF. O ser parturiente: um enfoque vivencial. Niterói: UFF; 1998.

9. Neme B. Obstetrícia básica. 2a ed. São Paulo: Sarvier, 2000.

10. Soler ZASG. Estudo do quarto período do parto: uma proposta de assistência sistematizada de enfermagem [tese]. São Paulo: Escola de Enfermagem da Universidade de São Paulo; 1993.

11. Rezende J. Obstetrícia. 9a ed. Rio de Janeiro: Guanabara Koogan; 2003.

12. Ruiz MT, Soler ZASG. Características do atendimento da equipe obstétrica à mulher durante o quarto período do parto em um hospital geral de ensino. Enfermagem Brasil; 2007;6(4):248-55.

13. Lowdermilk DL, Perry SE, Bobak IM. O cuidado em enfermagem maternal. 5a ed. Porto Alegre: Artmed; 2002.

14. Lowe NK. The nature and management of labor pain: peer-reviewed papers from an evidence-based symposium. Am J Obstet Gynecol 2002;186(5): S1-15.

15. Mamede FV, Almeida AM, Souza L, Mamede MV. Pain during the labor active phase: the effect of walking. Rev Latinoam Enfermagem 2007;15(6):1157-62.

16. North American Nursing Diagnosis Association. Diagnósticos de enfermagem da NANDA: definiçóes e classificação 2005- 2006. Porto Alegre: Artemed; 2006.

17. Mota DDCF, Cruz DALM, Pimenta CAM. Fadiga: uma análise do conceito. Acta Paul Enferm 2005;18(3):285-93.

18. Murtagh J. Fatigue - a general diagnostic approach. Aust Fam Phys 2003,32(11):873-6.

19. Dick ML, Sundin J. Psychological and psychiatric causes of fatigue - assessment and management. Aust Fam Phys 2003;32(11):877-81. 
20. Alves VM, Moura ZA; Palmeira ILT; Lopes MVO. Estudo do diagnóstico de enfermagem fadiga em gestantes atendidas numa unidade básica de atenção à saúde. Acta Paul Enferm 2006;19(1):70-5.

21. Alvim DAB, Bassoto TRP, Marques GM. Sistematização da assistência de enfermagem à gestantes de baixo risco. Rev Meio Amb Saúde 2007;2(1):258-72.
22. Brasil. Ministério da Saúde. Secretaria de Atenção à Saúde. Departamento de Ações Programáticas. Área Técnica de Saúde da Mulher. Pré-Natal e Puerpério: atenção qualificada e humanizada - manual técnico. Brasília: Ministério da Saúde; 2005.

23. Duarte G, Cunha SP, Mauad Filho F, Nogueira AA, Berezowski AT, Rodrigues R. Protocolos de condutas em gestação de alto risco. Ribeirão Preto: Funpec; 2003. 